\title{
CAPACIDADE DE SOBREVIVÊNCIA DO EMBRIÃO DE Rhipicephalus microplus (CANESTRINI, 1887) (ACARI: IXODIDAE) A ALTERAÇÕES BRUSCAS DE TEMPERATURA
}

\author{
Michele Costa Pinheiro1, Iwine Joyce Sá-Hungaro¹, Camila Dantas Martins², \\ Vanessa Almeida Raia1, Kátia Maria Famadas ${ }^{1}$ \\ 1 UFRRJ \\ 2 UNESA \\ Correspondência: Michele Costa Pinheiro: michelezootec@gmail.com
}

\begin{abstract}
RESUMO: A embriogênese é um dos eventos chave no desenvolvimento dos artrópodes, principalmente quando ela ocorre no ambiente, como é o caso dos carrapatos. Dentre os fatores abióticos que influenciam no ciclo de vida desses artrópodes, a temperatura parece ser o mais importante. Na Baixada Fluminense já foram registradas pela Estação Metereológica da Pesagro-Rio flutuações significativas na temperatura diária. Justifica-se então elucidar o comportamento do embrião de Rhipicephalus microplus frente às variações de temperatura. Para tal foram realizados experimentos em condições controladas de laboratório $\left(27^{\circ} \pm 1^{\circ} \mathrm{C}\right.$ e $80 \pm 10 \%$ UR), nos quais embriões no $15^{\circ}$ dia de incubação foram submetidos a diferentes tempos de exposição em temperaturas extremas. O período de incubação dos ovos de $R$. microplus usados neste estudo foi de $23 \pm 0,78$ dias. Em todos os tratamentos foi possível observar a presença do saco retal através da microscopia óptica. Pode-se concluir que embriões de $R$. microplus expostos por diferentes períodos de temperaturas tem a capacidade de suportar pelo menos 36 horas a $18^{\circ} \mathrm{C}$ ou $32^{\circ} \mathrm{C}$, temperaturas estas, abaixo da média mínima e acima da média máxima encontrada na região, sem que estas sejam deletérias ao embrião.
\end{abstract}

Palavras-chave: carrapatos; desenvolvimento embrionário; mudanças climáticas; saco retal

\section{CAPACITY OF SURVIVAL OF EMBRYO Rhipicephalus microplus (CANESTRINI, 1887) (ACARI: IXODIDAE) UNDER SUDDEN CHANGES OF TEMPERATURE}

\begin{abstract}
Embryogenesis is a key event in the development of arthropods, especially when it occurs in the environment, such as ticks. Among the abiotic factors that influence the life cycle of these arthropods, the temperature seems to be the most important. Baixada Fluminense has been recorded by the Meteorological Station of Pesagro -Rio significant fluctuations in daily temperature. Justified then elucidate the behavior of Rhipicephalus microplus embryo to variations in temperature. For this purpose experiments were performed under controlled laboratory conditions ( $27 \pm 1 \stackrel{\circ}{C}$ and $80 \pm 10 \% \mathrm{RH}$ ), in which embryos on the 15th day of incubation were subjected to different exposure times at extremes of temperature. The incubation period of eggs of $R$. microplus used in this study was $23 \pm 0.78$ days. In all treatments was possible to observe the presence of rectal bag by optical microscopy. It can be concluded that embryos R. microplus exposed for different periods of temperatures has the capability of supporting at least 36 hours at $18^{\circ} \mathrm{C}$ and $32^{\circ} \mathrm{C}$, these temperatures, below minimum and above the maximum average found in the average region without these are harmful to the embryo.
\end{abstract}

Key Words: climate change; embryonic development; rectal sac; ticks 


\section{INTRODUÇÃO}

Dentre os ixodídeos que parasitam animais domésticos, Rhipicephalus microplus (Canestrini, 1887) tem expressão significativa sobre aumento do custo na produção de bovinos no Brasil. Tal fato se deve aos prejuízos que gera (Grisi et al., 2002; Leal et al., 2003).

A distribuição de $R$. microplus entre os paralelos $32^{\circ}$ Norte e $32^{\circ}$ Sul tem como uma das justificativas fatores relacionados à temperatura ambiente. No Brasil, este carrapato encontra condições climáticas favoráveis ao seu desenvolvimento em regiões que vão do extremo Sul em direção ao Norte e Nordeste, possibilitando uma variação de 2,5 até 5 gerações por ano (Vidotto, 2002).

O ciclo de $R$. microplus se passa, na maior parte do tempo, sobre seu hospedeiro, ocorrendo no ambiente somente as fêmeas em oviposição, a embriogênese e larvas recém-eclodidas (Furlong, 1993). Assim métodos de controle têm sido empregados visando a eliminação do parasitismo sobre o hospedeiro e a redução das formas infestantes, no ambiente (Santos Junior et al., 2000).

No que se refere aos estágios de vida livre, para implementação de métodos de controle estratégico, principalmente aqueles que visem à redução das larvas infestantes nas pastagens, é necessário conhecimento amplo sobre ecologia, no que concernem às variações climáticas e microclimáticas. Nesse aspecto, temperatura e umidade são as duas variáveis abióticas com maior influência sobre as fases de vida livre dos carrapatos em geral (Buczek, 2000; Lindgren et al., 2000; Gray, 2002). O conhecimento do microclima é, portanto, estratégico uma vez que as variações de temperatura e umidade podem influenciar na quantidade de larvas na pastagem, modificando assim todo 0 panorama do controle (Furlong e Prata, 2005).

Sob o ponto de vista da fisiologia, temperaturas elevadas são responsáveis pela aceleração no metabolismo dos artrópodes e consequentes reduções no tempo entre gerações. Em contraposição, baixas temperaturas promovem redução nas taxas metabólicas e nas fases de vida livre do $R$. microplus foram responsabilizadas por interferir negativamente causando prolongamento no período de incubação e/ou mesmo redução na eclodibilidade das larvas (Londt, 1977; Buczek, 1987; Glória et al., 1993).

A embriogênese nos carrapatos se inicia quando o ovo ganha o ambiente (Soneshine, 1991). Uma das estruturas que sustenta a hipótese de que a embriogênese teve seu curso normal, é o saco retal, que somado aos túbulos de Malphigi se destaca na figura do ovo devido ao seu aspecto branco leitoso, que é resultante do acúmulo de grânulos de guanina, um metabólito produzido pelo embrião. Assim a presença da guanina indica plena atividade do embrião (Jasik e Buczek, 2005).

Embora os efeitos da temperatura sobre a postura e taxa de eclodibilidade larval para ixodídeos do gênero Rhipicephalus estejam bem elucidados (Hitchcock, 1955; Dipeolu, 1984; Davey e Cooksey, 1989; Dantas-Torres et al., 2010), pouco ainda se sabe dos impactos que oscilações de temperatura podem causar sobre o desenvolvimento embrionário. Cabe destacar que como espécie de ampla distribuição geográfica $R$. microplus, pode expressar adaptações às adversidades climáticas em cada área de sua distribuição (Famadas e Faccini, 1989; Passos et al., 1999; Ullmann et al., 2005). Somese a isso que a diversidade de 
ambientes, sistemas produtivos e condições climáticas são capazes de exercer pressões significativas sobre essas populações e, portanto sobre a biologia do carrapato do boi. Desta forma a investigação sobre diferentes populações de carrapatos ao longo de sua área de distribuição é de suma importância.

Pesquisas sobre bioecologia de carrapatos de animais domésticos e silvestres vêm sendo desenvolvidas nos Laboratórios de Ixodologia e Morfofisiologia de Ácaros do Departamento de Parasitologia Animal da Universidade Federal Rural do Rio de Janeiro, há vários anos, e este estudo teve como objetivo desafiar a embriogênese de uma população de $R$. microplus frente a diferentes períodos de exposição em temperaturas extremas em condições de laboratório.

\section{MATERIAL E MÉTODOS}

\section{Origem e Manutenção de Rhipicephalus microplus}

Os ixodídeos foram provenientes da segunda geração de uma colônia de $R$. microplus originária de fêmeas coletadas em bovinos no município de Seropédica (latitude 22\%44'38" Sul e longitude $43^{\circ} 42^{\prime} 27^{\prime \prime}$ Oeste, altitude $26 \mathrm{~m})$. Os carrapatos foram mantidos por infestação artificial em bovinos mestiços livres de doenças e sem contato com carrapaticidas. Os bovinos foram mantidos alimentados com ração própria para a espécie e capim-branquiária sem contato prévio com infestação de carrapatos e água ad libitum. No laboratório as fêmeas obtidas de queda natural foram limpas com solução de hipoclorito a $1 \%$, secadas, pesadas em balança analítica e distribuídas nos grupos experimentais de forma em que 0 peso das fêmeas fosse semelhante. Formados os grupos experimentais as fêmeas foram individualizadas pela fixação com esparadrapos em decúbito dorsal em placas de Petri (150x200mm) que foram mantidas em estufa biológica tipo BOD (Biological Oxigen Demand) regulada a $27 \pm 1^{\circ} \mathrm{C}, 80 \pm 5 \%$ UR, escotofase.

\section{Procedimento Experimental}

Quatrocentas e cinquenta teleóginas com peso médio de 265,7 \pm 41,6 mg foram distribuídas em 9 grupos com 50 fêmeas cada. Os tratamentos estudados foram $18^{\circ} \mathrm{C}$ e $32^{\circ} \mathrm{C}$ durante 6 , 12,24 e 36 horas e $27^{\circ} \mathrm{C}$ como tratamento controle.

Ovos de terceiro dia de postura foram utilizados sendo misturados e organizados nas unidades experimentais (temperatura/tempo de exposição) e novamente mantidos em BOD a $27^{\circ} \mathrm{C}$.

No $15^{\circ}$ dia cada unidade experimental foi alocada nas temperaturas de 18 e $32^{\circ} \mathrm{C}$ pelos respectivos tempos de exposição $(6,12$, 24 e 36 horas), retornando posteriormente para a temperatura de $27^{\circ} \mathrm{C}$.

Depois de submetidos aos tratamentos, já retornados a temperatura controle, a cada 24 horas foi coletada uma alíquota de $8 \mathrm{mg}$ de ovos de cada unidade experimental, até a eclosão da primeira larva ou que fosse constatada a inviabilidade dos ovos, por seu aspecto escuro e desagregado. As alíquotas de ovos foram colocadas em tubos tipo Eppendorf $\AA$ com etanol $70 \%$ para observação do embrião a posteriori.

\section{Preparo e montagem dos ovos}

Os ovos de $R$. microplus fixados foram clarificados por 24 horas em lactofenol e novamente retornados para álcool $70^{\circ} \mathrm{GL}$. Os ovos foram então, montados temporariamente com álcool $70^{\circ} \mathrm{GL}$ em lâmina escavada coberta por lamínula para o exame do saco retal, estrutura resultante do desenvolvimento embrionário (DIPEOLU, 1984). 


\section{Análise Estatística}

A análise das porcentagens para presença de saco retal entre as temperaturas e tempos foi realizada pelo teste Anova e quando necessário por Tukey-Kramer com o programa computacional $\mathrm{R}$, ao nível de $5 \%$ de significância.

\section{RESULTADOS}

O período médio de incubação observado nos grupos experimentais foi de $23 \pm 0,78$ dias. Em todos os tratamentos foi possível observar a presença do saco retal (SR) durante todo o período experimental.

Ovos do grupo controle, mantidos constantemente a $27^{\circ} \mathrm{C}$, apresentaram período de incubação de 23 dias com $94,41 \%$ de presença do SR na massa de ovos antes da eclosão da primeira larva.

Ovos mantidos a $18^{\circ} \mathrm{C}$ durante 6 , 24 e 36 horas, tiveram seu período de incubação de 24 dias com 94,35; 95,89 e $94,67 \%$ dos ovos com SR, respectivamente. No tratamento $18^{\circ} \mathrm{C}$ durante 12 horas, 96,06\% dos ovos apresentavam saco retal ao final do período de incubação, que foi de 23 dias (Figura 1).

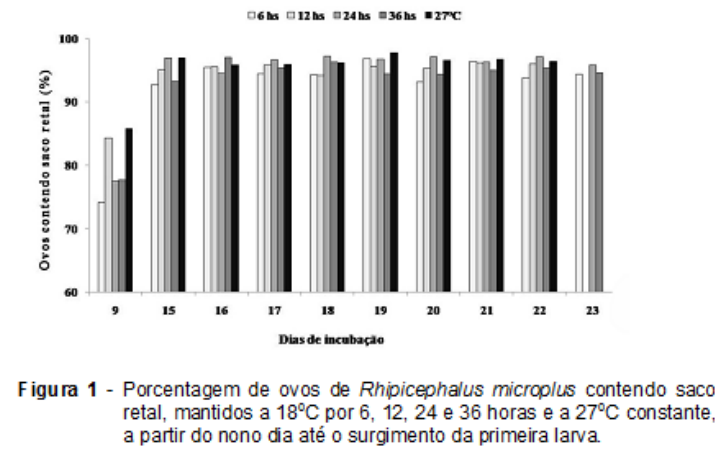

Nos tratamentos $32^{\circ} \mathrm{C}$ durante 6 e 12 horas o período de incubação foi de 23 dias, com 96,29 e 93,57\% dos ovos com saco retal, respectivamente. Esse período foi mais curto, 22 dias, nos tratamentos $32^{\circ} \mathrm{C}$ por 24 horas

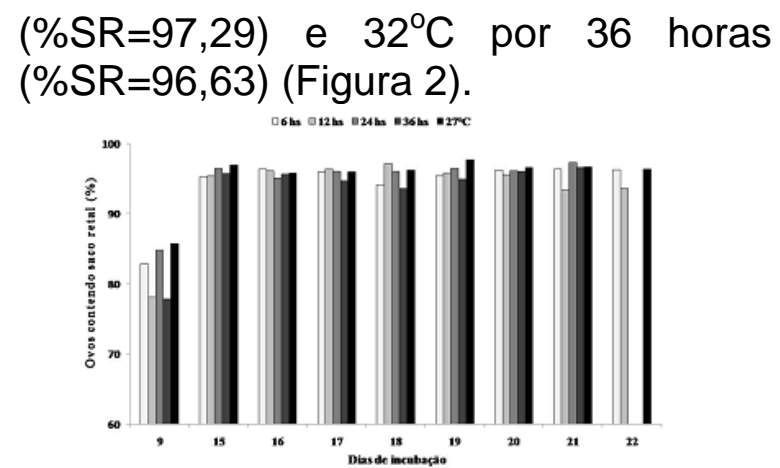

Figura 2 - Porcentagem de ovos de Rhipicephalus microp/us contendo saco retal, mantidos a $32^{\circ} \mathrm{C}$ por $6,12,24$ e 36 horas, e a $27^{\circ} \mathrm{C}$ constante, a partir do nono dia até o surgimento da primeira larva

Quando os percentuais de presença do saco retal nos diferentes tempos de exposição foram comparados dentro de cada temperatura não foram observadas diferenças $(P>0,05)$. No entanto, quando todas as temperaturas e tempos de exposição foram comparadas observou-se diferença no tratamento $32^{\circ} \mathrm{C}$ pelo período de 36 horas, quando comparado aos de $18^{\circ} \mathrm{C} /$ 24 horas; grupo controle $\left(27^{\circ} \mathrm{C}\right.$ constante); e $32^{\circ} \mathrm{C} / 24$ horas $(\mathrm{P}<0,05)$.

Não foram observados encarquilhamento, desagregação ou qualquer anormalidade nos ovos em nenhum dos tratamentos.

\section{DISCUSSÃO}

Vale esclarecer que as temperaturas escolhidas, $18^{\circ} \mathrm{C}$ e $32^{\circ} \mathrm{C}$, são consideradas próximas à média mínima e a média máxima registrada no município de Seropédica, onde segundo a estação meteorológica da PESAGRORJ e Instituto Nacional de Meteorologia (INMET), a temperatura média anual dos últimos 20 anos foi de $23,83^{\circ} \mathrm{C}$ e as temperaturas médias anuais mínimas e máximas são, respectivamente, $20,1^{\circ} \mathrm{C}$ e $29,8^{\circ} \mathrm{C}$ (Figura 3). Décimo quinto dia de postura foi utilizado, pois segundo Carvalho (dados não publicados) $50 \%$ dos ovos analisados apresentaram embrião (= presença de saco retal). 


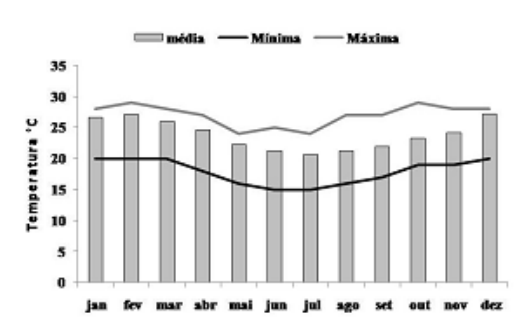

Figura 3 - Temperaturas média, máxima e mínima encontradas no município de Seropédica, Rio de Janeiro nos últimos 20 anos (Fonte: Pesagro-RJ)

A temperatura é um fator crucial no curso do desenvolvimento embrionário de ixodídeos (Buczek, 1987). Isso já foi comprovado em diversos experimentos, como o de Brovini et al., (2003), no qual os autores observaram que nas fêmeas de $R$. microplus, parâmetros relacionados ao ingurgitamento e o período de pré-postura e incubação são diretamente influenciados pela temperatura. Com exceção de regiões onde os invernos são rigorosamente frios, chegando a temperaturas muito baixas por longos períodos como apresentado nos trabalhos de Hitchcock (1955), Davey e Cooksey (1989), Dantas-Torres et al., (2010), a temperatura é um fator dinâmico e não permanece alta ou baixa por um período de tempo considerável. Essa mesma característica é observada na Baixada Fluminense, onde ao longo de 24 horas foram registradas flutuações significativas da temperatura, com variações de 5 a $10^{\circ} \mathrm{C}$ em um mesmo Embora considerada

relativamente baixa para região dos trópicos, os ovos submetidos a temperatura mínima de $18^{\circ} \mathrm{C}$ tiveram sua embriogênese completa, independente do período de exposição de $6,12,24$ e 36 horas. Em estudos realizados com outros estágios de vida de $R$. microplus e Rhipicephalus sanguineus da mesma região (Glória et al., 1993, Bellato e Daemon, 1997), foi observado um prolongamento do ciclo quando os carrapatos foram submetidos a temperatura de $18^{\circ} \mathrm{C}$ constante. Assim a redução da temperatura de $27^{\circ} \mathrm{C}$ para $18^{\circ} \mathrm{C}$ é capaz de promover desaceleração no desenvolvimento do embrião sem ser deletéria.

Espécies de carrapatos de climas temperados, como os observados por Hitchcock (1955), têm que lidar com uma variação sazonal ainda mais acentuada. Esse autor observou que na temperatura constante de $15^{\circ} \mathrm{C}$ por 16 dias, não houve desenvolvimento embrionário de $R$. microplus, no entanto quando os ovos foram transferidos para a temperatura de $25^{\circ} \mathrm{C}$ a embriogênese teve seu curso normal. Isso pode ser considerado uma evidência de que a temperatura é o fator condicionante para a ocorrência da diapausa morfogenética dos carrapatos, que segundo Randolph (2004), é uma adaptação utilizada por algumas espécies para garantir que a emergência das larvas coincida com as condições ótimas para sobrevivência. Vale destacar que temperaturas extremamente baixas por longos períodos podem ser deletérias para 0 embrião de algumas espécies de Rhipicephalus como observado para $R$. sanguineus por Dantas-Torres et al.,(2010).

Quando expostos a $32^{\circ} \mathrm{C}$, os ovos de $R$. microplus apresentaram eclosão larval em todos os tempos de exposição, e houve uma relação inversamente proporcional com 0 período de incubação, ou seja, quanto maior o tempo de exposição, menor o período de incubação (Figura 2). Embora esta temperatura não seja a máxima registrada na região, muitas espécies de carrapato são prejudicadas quando expostas constantemente a ela durante a embriogênese, como é o caso de $R$. sanguineus (Bellato e Daemon, 1997) e Amblyomma cajennense (Chacón et al., 2003).

Os resultados alcançados com de $R$. microplus no que tange a exposição do embrião a $32^{\circ} \mathrm{C}$ por 36 horas, ou seja, redução no tempo de incubação, pode indicar que curtos períodos de exposição a altas temperaturas, neste 
caso $32^{\circ} \mathrm{C}$, podem ser benéficos para o desenvolvimento embrionário dos ixodídeo ou que $R$. microplus seja uma espécie mais adaptada a região. No entanto mais estudos devem ser realizados para verificação destas hipóteses.

Vale ressaltar que o período máximo estabelecido para a temperatura extrema de $32^{\circ} \mathrm{C}$ é passível de ocorrer na região, e que a despeito dos demais estudos realizados foi possível verificar se o embrião já formado sofre danos quando submetidos a este desafio.

Nenhuma alteração morfológica foi observada macroscopicamente e/ou microscopicamente na massa de ovos ou nos ovos clarificados, respectivamente. Buczek (2000) associou alterações observadas nos embriões e larvas de Hyalomma marginatum marginatum com a umidade relativa e temperatura próximas às utilizadas em nosso grupo controle, $90 \%$ e $25^{\circ} \mathrm{C}$. Suas amostras apresentaram deformidades durante a embriogênese e as larvas sobreviventes apresentaram alterações nos palpos, quelíceras e hipostômio.

Como relatado em estudos sobre R. microplus na América do Sul, este carrapato é mais encontrado em condições quentes e úmidas (Martins et al., 2002), essas condições afetam diretamente a fase não parasitária do ixodídeo, uma vez que é no ambiente onde estão sujeitos as intempéries do clima, pois na fase parasitária as condições de vida são constantes (Furlong e Prata, 2005).

Por isso é durante a fase em que o carrapato se encontra no campo que medidas estratégias de controle mais eficazes podem ser implementadas.

\section{CONCLUSÃO}

A população de $R$. microplus da Baixada Fluminense enquanto embrião não sofre danos caso a temperatura considerada adequada aos ixodídeos neotropicais $\left(27^{\circ} \mathrm{C}\right)$ sofra variações de menos $5^{\circ} \mathrm{C}$ ou mais $9^{\circ} \mathrm{C}$ por até 36 horas.

\section{AGRADECIMENTOS}

Este projeto teve o auxílio da Coordenação de Aperfeiçoamento de Pessoal de Nível Superior - CAPES. Os autores agradecem ao Laboratório de Quimioterapia Experimental em Parasitologia (Universidade Federal Rural do Rio de Janeiro) pela cessão das teleóginas usadas no estudo.

\section{REFERÊNCIAS}

BELLATO, V.; DAEMON, E. Efeito de três temperaturas sobre a fase não parasitária de Rhipicephalus sanguineus (Latreille, 1806) (Acari: Ixodidae). Revista Brasileira de Parasitologia Veterinária, v.6, n.1, p.21-27, 1997.

BROVINI, C.N.; FURLONG, J.; CHAGAS, A.C.S. Influência dos fatores climáticos na biologia e no comportamento de fêmeas ingurgitadas de Boophilus microplus a campo. Bioscince Journal, v.19, n.1, p.71-76, 2003.

BUCZEK, A. Studies on the Biology of $\operatorname{Argas}(A$.) refiexus Fabricius, 1794 (Acari:Ixodida:

Argasidae). 1. Effect of Temperature and Relative Humidity on Embryonic Development and Egg Hatch. Folia Biologica, v.36, n.3-4, p.238-264, 1987.

BUCZEK, A. Experimental Teratogeny in the Tick Hyalomma marginatum marginatum (Acari: Ixodida: Ixodidae): Effect of High Humidity on embryonic development. Journal Medical Entomology, v.37, n.6, p.807-814, 2000.

CHACÓN, S.C.; CORREIA, P.G.; BARBIERI, F.S. et al. Efeito de três temperaturas constantes sobre a fase não parasitária de Amblyomma cajennense (Fabricius, 1787) (Acari: Ixodidae). Revista Brasileira de Parasitologia Veterinária, v.12, n.1, p.13-20, 2003. 
DAVEY, R.B.; COOKSEY, L.M. Effects of prolonged exposure at low temperature on Boophilus microplus (Acari: Ixodidae). Journal Medical Entomology, v.26, n.5, p.407-410, 1989.

DANTAS-TORRES, F.D.; GIANNELLI, A.; FIGUEIREDO, L.A. et al. Effects of prolonged exposure to low temperature on eggs of the brown dog tick, Rhipicephalus sanguineus (Latreille, 1806) (Acari: Ixodidae). Veterinary Parasitology, v.171, n.3-4, p.327-330, 2010.

DIPEOLU, O.O. Studies on ticks of veterinary importance in Nigeria: X. Notes on the biology of ticks of dogs Rhipicephalus sanguineus and Haemaphisalis leachi leachi. Bulletin Of Animal Health and Production in Africa, v.32, n.1, 1984.

FAMADAS, K.M.; FACCINI, J.L.H. Variação morfológica de Boophilus microplus (Canestrini, 1887) (Acari: Ixodidae) no Brasil. Arquivos da Universidade Federal Rural do Rio de Janeiro, v.12, n.1-2, p.73-81, 1989.

FURLONG, J. Controle do carrapato dos bovinos na Região Sudeste do Brasil. Caderno Técnico Escola Veterinária UFMG, n.8, p.4961, 1993.

FURLONG, J.; PRATA, M.C. Conhecimento básico para o controle do carrapato-dosbovinos. In: FURLONG, J (Ed.). Carrapato: problema e soluções. Juiz de Fora: Embrapa Gado de Leite, 2005. p. 9-20.

GLÓRIA, M.A.; DAEMON, E.; FACCINI, J.L.H. et al. Influência de diferentes temperaturas sobre a biologia da fase não parasitária de Boophilus microplus (Canestrini, 1887) (Acari: Ixodidae). Revista Brasileira de Parasitologia Veterinária, v.2, n.2, p.85-91, 1993.

GRISI, L.; MASSARD, C.L.; MOYA-BORJA, G.E. et al. Impacto econômico das principais ectoparasitoses em bovinos no Brasil. Hora Veterinária, v.2, n.125, p.1:8-10, 2002.

HITCHCOCK, L.F. Studies on the non-parasitic stages of the cattle tick, Boophilus microplus (Canestrini) (Acarina Ixodidae). Australian Journal of Zoology, v.3, p.295-311, 1955.

Instituto Nacional de Meteorologia - INMET. Disponível em:

<http://www.inmet.gov.br/sim/gera_graficos.php ?chklist $=1 \% 2 \mathrm{C} 2 \% 2 \mathrm{C} \& U \mathrm{~F}=$ \&mostrar=1 \&imgmap $=\&$ Data $=07 \% 2 F 2009 \&$ Data2 $=2013 \&$ enviar $=$ Most rar+Gr\%E1ficos> Acesso em: 30/07/2013.

JASIK, K.; BUCZEK, A. Origin of alimentary tract in embryogenesis of Ixodes ricinus (Acari: Ixodidae). Journal Medical Entomology, v.42, n.4, p.541-547, 2005.
LEAL, A.T.; FREITAS, D.R.J.; VAZ JÚNIOR, I.S. Perspectivas para o controle do carrapato bovino. Acta Scientiae Veterinariae, v. 31, n. 1, p. 1-11, 2003.

LONDT, J.G.H. Oviposition and incubation in Boophilus decoloratus (Koch, 1844) (Acarina: Ixodidae). Onderstepoort Journal Veterinary Research, v.44, n.1, p.13-20, 1977.

MARTINS, J.R.; EVANS, D.E.; CERESÉR, V.H. et al. Partial strategic control within a herd of European breed cattle in the state of Rio Grande do Sul, southern Brazil. Experimental Applied Acarology, v.27, n.3, p.241-251, 2002.

PASSOS, D.T.; FERREIRA, C.A.S.; SILVA, S.S. et al. Detection of genomic variability in different populations of the cattle tick Boophilus microplus in southern Brazil. Veterinary Parasitology, v.87, n.1, p.83-92, 1999.

RANDOLPH, S.E. Tick ecology: processes and patterns behind the epidemiological risk posed by ixodid ticks as vectors. Parasitology, v.129, p.37-65, 2004.

SANTOS JÚNIOR, J.C.B.; FURLONG, J.; DAEMON, E. Controle do carrapato Boophilus microplus (Acari: Ixodidae) em sistemas de produção de leite da microrregião fisiográfica fluminense do Grande Rio - Rio de Janeiro. Ciência Rural, v. 30, n. 2, p. 305-311, 2000.

SONESHINE, D.E. Biology of ticks. v.1. Oxford University Press, New York, 1991, 447pp.

ULLMANN, A.J.; LIMA, C.M.R.; GUERRERO, F.D. et al. Genome size and organization in the blacklegged tick, Ixodes scapularis and the Southern cattle tick, Boophilus microplus. Insect Molecular Biology., v. 14, n.2 p.217-222, 2005.

VIDOTTO, O. [2002]. Complexo Carrapato Tristeza parasitária e outras parasitoses de bovino. Disponível em:

http://www.nupel.uem.br/pos-ppz/complexo-0803.pdf. Acesso em 23/03/2013. 